Document downloaded from:

http://hdl.handle.net/10251/50838

This paper must be cited as:

Pitarch Pérez, JL.; Sala Piqueras, A. (2014). Multicriteria fuzzy-polynomial observer design for a 3DoF nonlinear electromechanical platform. Engineering Applications of Artificial Intelligence. 30:96-106. doi:10.1016/j.engappai.2013.11.006.

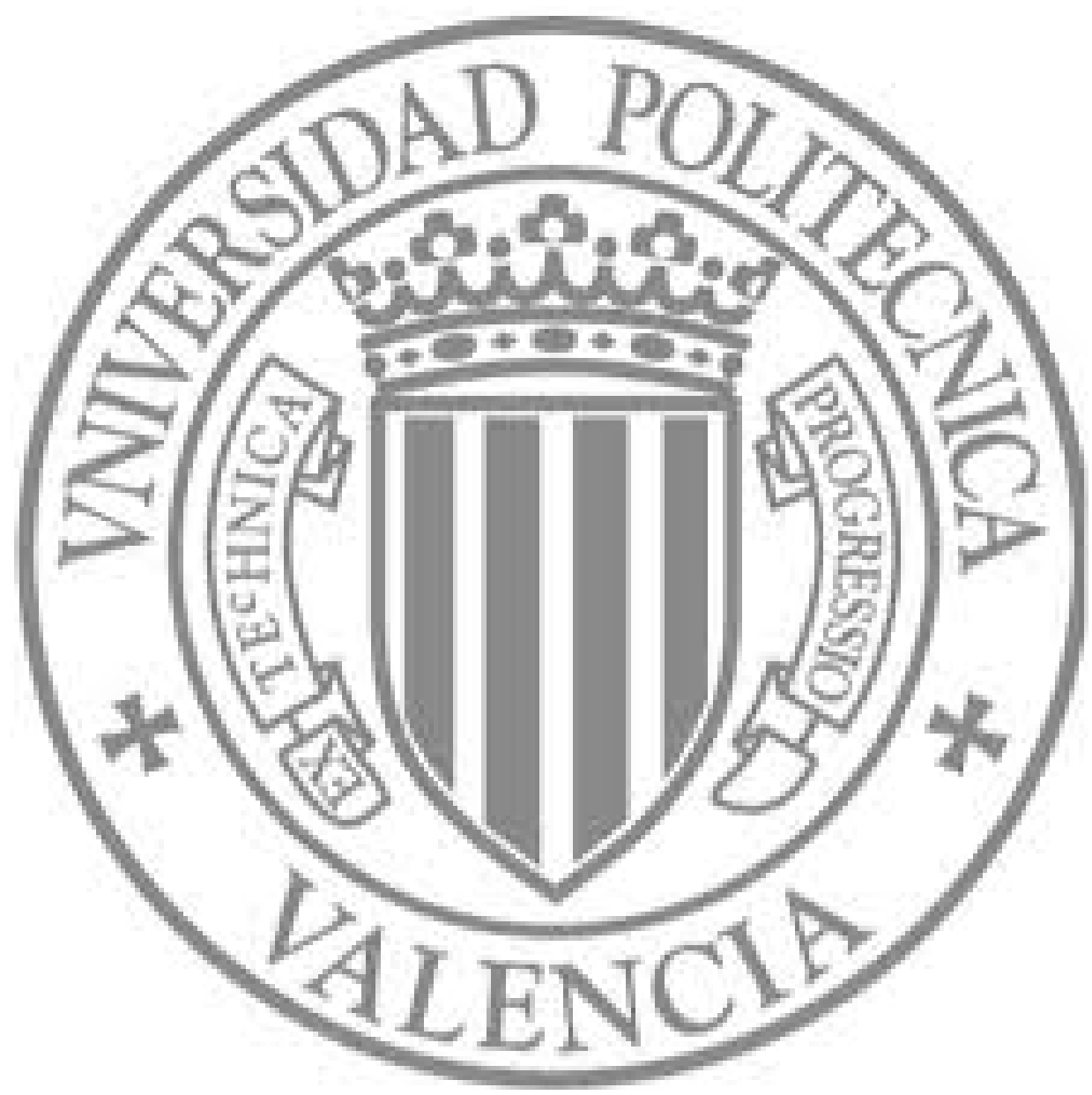

The final publication is available at

http://dx.doi.org/10.1016/j.engappai.2013.11.006

Copyright Elsevier 


\title{
Multicriteria fuzzy-polynomial observer design for a 3 DoF nonlinear electromechanical platform
}

\author{
J.L. Pitarch ${ }^{\mathrm{a}, *}$, A. Sala ${ }^{\mathrm{a}}$ \\ ${ }^{a}$ Systems Engineering and Control Department (ISA), Instituto de Automatica e Informatica \\ Industrial (AI2), Universitat Politècnica de Valencia.
}

\begin{abstract}
This paper proposes local fuzzy-polynomial observer discrete-time designs for state estimation of a nonlinear 3 DoF electromechanical platform (fixed quadrotor). A trade-off between $\mathcal{H}_{\infty}$ norm bounds and speed of convergence performance is taken into account in the design process. Actual experimental data are used to compare performance of the fuzzy polynomial design with classical ones based on the Takagi-Sugeno and linearized models, both using the same optimization criteria and design parameters.
\end{abstract}

Keywords: fuzzy polynomial systems, multicriteria optimisation, Sum of Squares, $\mathcal{H}_{\infty}$ attenuation, state estimation, electromechanical systems

\section{Introduction}

In many cases, nonlinear systems can be modelled as fuzzy systems which interpolate between linear [1] or polynomial vertex models [2, 3]. Fuzzy-sector modelling is still nowadays a successful technique for the control of such class of systems $[4,5]$. Fuzzy systems with linear consequents are denoted as Takagi-Sugeno ones (TS), and those with polynomial consequents are denoted as polynomial fuzzy systems.

Polynomial fuzzy systems have been proved to be amenable to direct analysis with convex optimization, by using sum of squares (SOS) tools [6]; such tools transform SOS problems as semidefinite programming (SDP) problems, and then call on widely-used standard linear matrix inequality (LMI) solvers.

State estimators are important in control applications because most fuzzy control laws in literature require knowledge of the state for its implementation. The design of state observers for nonlinear systems using fuzzy TS models has been actively considered recently in practical control applications, [7, 8, 9].

This paper extends the above literature to the polynomial framework, allowing to obtain better results due to the lower mismodelling possible with polynomial equations. Furthermore, in order to get sensible solutions, the paper discusses a practical design which proposes a tradeoff between tracking speed versus $\mathcal{H}_{\infty}$ worst-case attenuation in a multicriteria setting.

\footnotetext{
* Corresponding author

Email addresses: jopipe1@upvnet.upv.es (J.L. Pitarch), asala@isa.upv.es (A. Sala) Preprint submitted to Engineering Applications of Artificial Intelligence 
In order to demonstrate the results, this paper considers application of such techniques to angular speed estimation on a 3 doF electromechanical system with noisy sensors, reporting a detailed comparative analysis between fuzzy and non-fuzzy (linearised) techniques.

The objective of this paper is designing a discrete-time polynomial observer with low computational cost in implementation, for a mechatronic system and validating it with experimental data. The concrete goals are:

1. Developing multiobjective $\mathcal{H}_{\infty}$ plus decay-rate observers for fuzzy polynomial systems, relaxing conservativeness by adding local information.

2. Adding extra constraints regarding the fastest components of the dynamics, in order to avoid undesirable behaviours in practice due to approximate discretization.

3. Proposing a practical methodology to choose from the Pareto-front solutions.

4. Comparing the achieved results with other techniques in literature (with theoretical guarantees absent in linearised designs for nonlinear systems), and validating the results on an experimental benchmark.

This paper is structured as follows: Section 2 presents the experimental platform (quadrotor) and its approximate polynomial model; Section 3 presents the discrete-time polynomial observer design with dual decay-rate and $\mathcal{H}_{\infty}$ objective; Section 4 discusses the many compromises needed to craft a successful engineering application; Section 5 presents a comparative analysis between linear, Takagi-Sugeno and polynomial observers, both theoretically and experimentally. Finally, a conclusion section closes the paper.

\section{System description and modelling}

The experimental platform chosen to evaluate the performance of the designed state observer is a three degrees of freedom (3DoF) system provided by Quanser ${ }^{\mathrm{TM}}[10]$. The platform, shown in Fig. 1, consists in a quadrotor mounted on a 3 DoF pivot joint, such that the body can freely move in roll, pitch and yaw. The data acquisition tasks and the implementation of required control algorithms were carried out in a PC running Linux-RT on top of an Ubuntu 12.04 installation. The communications between the quadrotor platform and the $\mathrm{PC}$ were made with a PMC I/O board. The sensors of the platform are encoders that measure the position of the three orientation-axes of the quadrotor $\phi, \theta$ and $\psi$. The control inputs are the voltages $V_{1}, V_{2}, V_{3}$ and $V_{4}$ applied to each of the 4 propellers of the quadrotor. High-resolution encoders are available to estimate the "true" speeds, but intentionally, a random noise has been added to them in order to evaluate the behavior with much lower-quality sensors in a more demanding setting. Indeed, the objective of the paper is testing the differences between linear and polynomial approaches in far-from-ideal cases: as expected from common sense, if sensors were "excellent" then any optimal observer design would, basically, disregard the (more uncertain) model equations.

An approximate non-linear model of the $3 \mathrm{DoF}$ platform is presented in the following equations giving accelerations in roll, pitch and yaw coordinates, as given in [11]: 


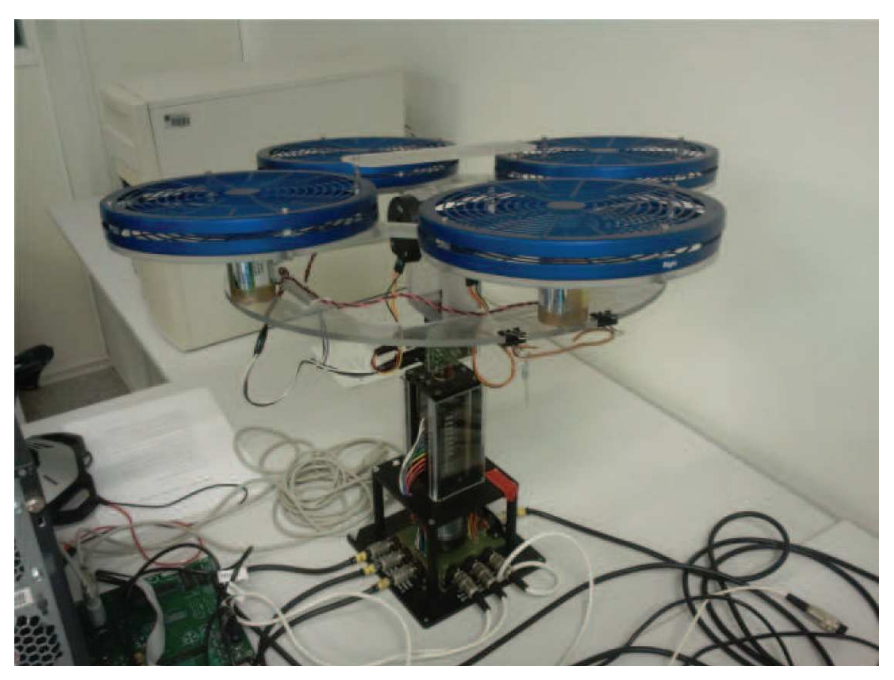

Figure 1: The Quanser quadrotor 3DoF system.

$$
\begin{aligned}
& \ddot{\phi}=\frac{J_{r} \dot{\theta}}{I_{x x}} u_{g}+\frac{I_{y y}-I_{z z}}{I_{x x}} \dot{\theta} \dot{\psi}+u_{1} \\
& \ddot{\theta}=\frac{J_{r} \dot{\phi}}{I_{x x}} u_{g}+\frac{I_{z z}-I_{x x}}{I_{y y}} \dot{\psi} \dot{\phi}+u_{2} \\
& \ddot{\psi}=\frac{I_{x x}-I_{y y}}{I_{z z}} \dot{\theta} \dot{\phi}+u_{3}
\end{aligned}
$$

where the gyroscopic effects in the roll and pitch dynamics contain the term

$$
u_{g}\left(V_{1}, \ldots, V_{4}\right)=K_{v}\left(V_{1}+V_{3}-V_{2}-V_{4}\right)
$$

which is the sum of the applied (known) voltages. Furthermore, each acceleration input $\left(u_{1}, u_{2}, u_{3}\right)$ from the nonlinear propellers' actuation, depends on the applied voltages as follows ${ }^{1}$ :

$$
\begin{aligned}
& u_{1}=\frac{b l K_{v}^{2}\left(V_{2}^{2}-V_{4}^{2}\right)}{I_{x x}} \\
& u_{2}=\frac{b l K_{v}^{2}\left(V_{3}^{2}-V_{1}^{2}\right)}{I_{y y}} \\
& u_{3}=\frac{d K_{v}^{2}\left(V_{1}^{2}-V_{2}^{2}+V_{3}^{2}-V_{4}^{2}\right)}{I_{z z}}
\end{aligned}
$$

The symbols used and their values, where applicable, are given in Appendix A, Table A.3 (extracted from [10]). For observer design purposes, in what follows, the input voltage signals will be considered to be the transformed signals $u_{i}$.

\footnotetext{
${ }^{1}$ The square is shorthand for $\operatorname{sign}\left(V_{i}\right) V_{i}^{2}$ to account for upwards and downwards thrust.
} 


\subsection{Fuzzy modelling}

Fuzzy-polynomial model. The bounds on the term $u_{g}$ can be computed based on the bounds of the voltage input, and they are a lower bound $u_{g}=4 K_{v} V_{\min }$ and an upper one $\overline{u_{g}}=4 K_{v} V_{\max }$. Following well-known procedures [12], if we define the weighting functions

$$
\begin{gathered}
\mu_{1}\left(V_{1}, \ldots, V_{4}\right)=\frac{\overline{u_{g}}-u_{g}\left(V_{1}, \ldots, V_{4}\right)}{\overline{u_{g}}-\underline{u_{g}}} \\
\mu_{2}\left(V_{1}, \ldots, V_{4}\right)=1-\mu_{1}\left(V_{1}, \ldots, V_{4}\right)
\end{gathered}
$$

then, the term $u_{g}$ is expressed as $u_{g}=\mu_{1} u_{g}+\mu_{2} \overline{u_{g}}$. Note that the arguments to $\mu_{i}$ and $u_{g}$ are omitted for brevity.

With the above input voltage bounds and taking $x=(\phi, \dot{\phi}, \theta, \dot{\theta}, \psi, \dot{\psi})$ as states, denoting as premise variables $z(t)=\left(V_{1}, \ldots V_{4}\right)$, the quadrotor model (1) can be expressed at sample time $T_{s}=5 \mathrm{~ms}$ (by Euler-discretization method) in the form:

$$
\begin{aligned}
x_{k+1} & =\sum_{i=1}^{2} \mu_{i}\left(z_{k}\right) \mathcal{A}_{i}\left(x_{k}\right)+\mathcal{B}\left(u_{k}\right)+T_{s} Q w_{k} \\
y_{k} & =C\left(x_{k}\right)+R \nu_{k}
\end{aligned}
$$

where $x_{k}, z_{k}$ denote the samples $x\left(k T_{s}\right), z\left(k T_{s}\right)$, model matrices are given by

$$
\begin{gathered}
\mathcal{A}_{1}\left(x_{k}\right)=\left[\begin{array}{c}
x_{1_{k}}+0.005 x_{2_{k}} \\
x_{2_{k}}+0.012 x_{4_{k}}-0.005 x_{4_{k}} x_{6_{k}} \\
x_{3_{k}}+0.005 x_{4_{k}} \\
x_{4_{k}}-0.012 x_{2_{k}}+0.005 x_{2_{k}} x_{6_{k}} \\
x_{5_{k}}+0.005 x_{6_{k}} \\
x_{6_{k}}
\end{array}\right], \mathcal{A}_{2}(x)=\left[\begin{array}{c}
x_{1_{k}}+0.005 x_{2_{k}} \\
x_{2_{k}}-0.012 x_{4_{k}}-0.005 x_{4_{k}} x_{6_{k}} \\
x_{3_{k}}+0.005 x_{4_{k}} \\
x_{4_{k}}+0.012 x_{2_{k}}+0.005 x_{2_{k}} x_{6_{k}} \\
x_{5_{k}}+0.005 x_{6_{k}} \\
x_{6_{k}}
\end{array}\right], \\
\mathcal{B}\left(u_{k}\right)=\left[\begin{array}{c}
0 \\
0.005 u_{1_{k}} \\
0 \\
0.005 u_{2_{k}} \\
0 \\
0.005 u_{3_{k}}
\end{array}\right], C\left(x_{k}\right)=\left[\begin{array}{c}
x_{1_{k}} \\
x_{3_{k}} \\
x_{5_{k}}
\end{array}\right],
\end{gathered}
$$

$x \in \mathbb{R}^{6}$ is the state, $u \in \mathbb{R}^{3}$ is the control input, $y \in \mathbb{R}^{3}$ is the output and $w \in \mathbb{R}^{6}, \nu \in \mathbb{R}^{3}$ are unknown process disturbance inputs and measurement noise respectively. Matrices $Q$ and $R$ are scaling matrices for expected disturbance and noise powers respectively, later specified. In this way, we may assume that the norm of the vector $\left[w^{T} \nu^{T}\right]^{T}$ is 1 in later designs without loss of generality.

Definition 1. Systems expressed in the form (6) are denoted as fuzzy polynomial dynamic systems [2, 3], and matrices $\mathcal{A}_{i}$ are denoted as "vertex models".

The speeds of the quadrotor $\eta=(\dot{\phi}, \dot{\theta}, \dot{\psi})=\left(x_{2}, x_{4}, x_{6}\right)$ will be assumed to lie in the operating region:

$$
\Omega_{x}=\left\{x_{2}, x_{4}, x_{6}: \eta^{T} \eta \leq R_{\max }^{2}\right\},
$$

with $R_{\max }^{2}=3\left(\frac{\pi}{2}\right)^{2}$. The above bound include a hypercube centered in the origin spanning the interval $[-\pi / 2, \pi / 2]$ on each coordinate. 
Takagi-Sugeno model. In order to compare the experiment with existent results in TS fuzzy literature, the quadrotor dynamics (1) was also modeled as a TS system (linear consequents):

$$
\begin{aligned}
x_{k+1} & =\sum_{i=1}^{8} \mu_{i}\left(z_{k}\right) \mathcal{A}_{i} x_{k}+\mathcal{B} u_{k}+T_{s} Q w_{k} \\
y_{k} & =C x_{k}+R \nu_{k}
\end{aligned}
$$

The model had 8 vertices, because the Coriolis terms (products of angular speeds), say for instance $\dot{\phi} \dot{\theta}$, had to be modelled as a TS model interpolating between vertices $\mathbf{p} \dot{\theta}$ and $\mathbf{q} \dot{\theta}$ where $\mathbf{p}$ and $\mathbf{q}$ are constants denoting a priori chosen speed bounds $\dot{\phi}_{\text {max }}$ and $\dot{\phi}_{\text {min }}$, respectively; the resulting membership function depends on $\dot{\phi}$. Details on the model and procedure can be consulted in [8].

Linear model. In order to compare with standard linear time-invariant techniques, the linearized model of the quadrotor around $x=0$ is provided on Appendix A.

\section{Observer design for polynomial systems}

This section discusses the observer design for discrete-time systems expressed as fuzzy polynomial models.

The set of polynomials in a variable $z$ will be denoted as $\mathcal{R}_{z}$, and the n-dimensional vectors of polynomials as $\mathcal{R}_{z}^{n}$. The corresponding element of a polynomial symmetric expression will be denoted as $(*)$.

Polynomials in some variables $z$ which can be decomposed as a sum of squares [13] of other polynomials will be denoted by $\Sigma_{z}$. Similarly the $n \times n$ matrix SOS polynomials will be denoted by $\Sigma_{z}^{n \times n}$. Determining if a SOS decomposition of a polynomial exists is a convex problem. Ample information on SOS programming can be consulted in [6]; for convenience, some background material and results on the topic appear in Appendix B.

Definition 2. A fuzzy polynomial discrete-time observer for a fuzzy polynomial discretized system (6) is expressed as:

$$
\hat{x}_{k+1}=\sum_{i=1}^{r} \mu_{i}\left(z_{k}\right)\left(\mathcal{A}_{i}\left(\hat{x}_{k}\right)+L_{i}\left(\hat{x}_{k}, y_{k}\right) \cdot\left(y_{k}-\hat{y}_{k}\right)\right)+\mathcal{B}\left(u_{k}\right)
$$

where $r$ is the number of fuzzy rules and the observer gains $L_{i}$ may depend polynomially on sensor measurements and estimated states.

In the particular fuzzy polynomial model of the quadrotor (6), as the arguments of $\mu_{i}$ are measurable ( $z$ are the input voltages), on-line implementation of (9) can be carried out with very low computational requirements.

\subsection{Discrete-time design}

In order to carry out observer design, the observer error $e_{k}=x_{k}-\hat{x}_{k}$ follows the equation:

$$
e_{k+1}=\sum_{i=1}^{2} \mu_{i}\left(\mathcal{A}_{i}\left(x_{k}\right)-\mathcal{A}_{i}\left(\hat{x}_{k}\right)-L_{i}\left(\hat{x}_{k}, y_{k}\right)\left(C\left(x_{k}\right)+R \nu_{k}-C\left(\hat{x}_{k}\right)\right)\right)+T_{s} Q w_{k}
$$


Note that the well-known fact that linear systems are globally stable does not hold in the polynomial case; hence, many times the problems are infeasible if local regions $\left(\Omega_{x}\right.$, $\left.\Omega_{e}\right)$ are not introduced for states which do not appear linearly in the system's dynamics. In order to do that, the observer speed error $\left(e_{s}=\eta-\hat{\eta}\right.$ where $\hat{\eta}$ are the estimated speeds) is assumed to lie inside the same sphere as the actual speed, see (7), i.e.:

$$
\Omega_{e}=\left\{e_{s}^{T} e_{s} \leq R_{\max }^{2}\right\}
$$

This assumption is reasonable when the initial estimated state is $\hat{x}(0)=0$ (usual observer start-up condition) and the initial state is inside some level-set of a Lyapunov function (to be later computed): in that case, $e_{s}(0)=\eta(0)$ and stability will make the Lyapunov function decrease [14].

From the above considerations, on the following we will assume that the state and estimation error do not leave expected operation regions $e \in \Omega_{e}$ and $x \in \Omega_{x}$, being $\Omega_{e}$ and $\Omega_{x}$ described in (11) and (7) respectively. Also, the following notation will be used as a shorthand:

- $\overline{\mathcal{A}}_{i}\left(x_{k}, \hat{x}_{k}\right)$ stands as $\mathcal{A}_{i}\left(x_{k}\right)-\mathcal{A}_{i}\left(\hat{x}_{k}\right)$,

- likewise $\bar{C}\left(x_{k}, \hat{x}_{k}\right)$ stands as $C\left(x_{k}\right)-C\left(\hat{x}_{k}\right)$.

- Subscript notation of a sample $k$ is omitted for simplicity, i.e., $e$ stands as $e_{k}$ where appropriate.

Consider now a quadratic candidate Lyapunov function on the error in the form

$$
V(e)=e^{T} P e
$$

where $P$ is a constant symmetric positive-definite matrix.

Theorem 1. Given a decay rate parameter $\alpha>0$, a polynomial observer (9) which gives a $\mathcal{L}_{2}^{2}$ gain attenuation bound $\gamma$ on the estimation error for system (6) against the worst-case disturbance, over regions on the error (11) and the state (7), is obtained by solving the following SOS problem for $i=1,2$ :

Minimize $\gamma$ such that

$$
\begin{gathered}
x^{T} P x-\epsilon(x) \in \Sigma_{x} \\
{\left[\begin{array}{cc}
e^{T}\left(P-D^{T} D\right) e+\gamma W^{T} W-\Psi_{1 i}(\eta, \hat{\eta}) & (*)^{T} \\
P \overline{\mathcal{A}}_{i}(x, \hat{x})-H_{i}(\hat{x}, y) \bar{C}(x, \hat{x})+T_{s} P Q w-H_{i}(\hat{x}, y) R \nu & P
\end{array}\right] \in \Sigma_{x, \hat{x}, w, \nu}^{12 \times 12}} \\
{\left[\begin{array}{cc}
\exp \left(-2 \alpha T_{s}\right) e^{T} P e-\Psi_{2 i}(\eta, \hat{\eta}) & (*)^{T} \\
P \overline{\mathcal{A}}_{i}(x, \hat{x})-H_{i}(\hat{x}, C(x)) \bar{C}(x, \hat{x}) & P
\end{array}\right] \in \Sigma_{x, \hat{x}}^{12 \times 12}} \\
2 e^{T} P \overline{\mathcal{A}}_{i}(x, \hat{x})-2 e^{T} H_{i}(\hat{x}, C(x)) \bar{C}(x, \hat{x})-\Psi_{3 i}(\eta, \hat{\eta}) \in \Sigma_{x, \hat{x}}
\end{gathered}
$$

where $\Psi_{j i}(\eta, \hat{\eta})=s_{j i}(\eta, \hat{\eta})\left(R_{\max }^{2}-\eta^{T} \eta\right)+q_{j i}(\eta, \hat{\eta})\left(R_{\max }^{2}-e_{s}^{T} e_{s}\right)$ with $j=1,2,3$ to enforce validity in the regions of $\eta$ and $\hat{\eta}$ determined in (7) and $(11), \gamma>0, W=[w \nu]^{T}$ is the disturbance vector, $\left(s_{11}, s_{21}, q_{11}, q_{21}, s_{12}, s_{22}, q_{12}, q_{22}\right) \in \Sigma_{\eta, \hat{\eta}}$ are SOS multipliers, $\epsilon(x)$ is an arbitrary radially unbounded positive polynomial ${ }^{2}$ and $D$ is a constant matrix which

${ }^{2}$ In the implementation in Section $5, \epsilon(x)=10^{-4} x^{T} x$, for this theorem. 
stands as an user-defined scaling weight, in order to choose a particular combination of errors to minimize. The polynomial observer gains can be computed as $L_{i}(\hat{x}, y)=$ $P^{-1} H_{i}(\hat{x}, y)$.

If the above problem renders feasible, then:

1. If initial condition $e_{0}=0, \sqrt{\gamma}$ is an upper bound on the (weighted) $\mathcal{L}_{2}^{2}$ gain (which is the nonlinear analogous to the linear $\mathcal{H}_{\infty}$ norm) [15], i.e.:

$$
\sup _{0<\|w\|_{2}<\infty} \frac{\|D e\|_{2}^{2}}{\|W\|_{2}^{2}}<\gamma
$$

2. If the initial condition $e_{0} \neq 0$, the initial estimation error decays exponentially with rate $^{3} \alpha$ in absence of disturbances, i.e.:

$$
V\left(e_{k}\right) \leq\left(\exp \left(-2 \alpha T_{s}\right)\right)^{k} V\left(e_{0}\right)
$$

Proof. See Appendix C.

Remark 1. Conditions (16) are stated in order to avoid high-frequency oscillations with period $2 T_{s}$ in the error dynamics. Although such conditions may be considered as "optional", including them is reasonable in practice both for having an acceptable transient and to avoid exciting high-frequency dynamics in the underlying physical system: in this way the result will be more tolerant to errors (such as those due to Euler discretization and unmodelled dynamics). In TS and linear cases we get familiar pole-region LMIs, see later (23).

With some manipulations, existent results in fuzzy TS observer literature can be obtained as a particular case in a straightforward way. Note, however, that in the plant in consideration in this application paper, TS modelling has an important side effect: the observer problem gets converted to one with unmeasurable premises, as the memberships must be evaluated with speed estimates $\hat{\eta}$ instead of the actual ones $\eta$. This did not occur in the polynomial case, and it will lead to an important performance decrease as discussed in the experimental results (Section 5) and will require theoretical refinements, addressed next.

With the TS model (8), the observer error dynamics is given by

$$
e_{k+1}=\sum_{i=1}^{8} \mu_{i}\left(\hat{x}_{k}\right)\left(\left(\mathcal{A}_{i}-L_{i} C\right) e_{k}-L_{i} R \nu_{k}\right)+T_{s} Q w_{k}+\sum_{i=1}^{8}\left(\mu_{i}\left(z_{k}\right)-\mu_{i}\left(\hat{z}_{k}\right)\right) A_{i} x_{k}
$$

and the observer-model mismatch must fulfill a Lipschitz-like bound

$$
\left\|\sum_{i=1}^{8}\left(\mu_{i}(z)-\mu_{i}(\hat{z})\right) \mathcal{A}_{i} x\right\| \leq \sigma\|e\|
$$

in order to proceed further and set up LMI's (see $[8,9]$ ). The bound $\sigma$ depends on the shape of the memberships (actually, bounds in $\left\|\frac{\partial \mu}{\partial x}\right\|$ ), the model $\mathcal{A}_{i}$ and the modelling

\footnotetext{
${ }^{3} \alpha$ is a decay rate in continuous-time (exponential) equivalent units, i.e., the discrete (geometric) decay is $\exp \left(-\alpha T_{s}\right)$.
} 
region $\Omega_{x}$. For this quadrotor case, the taken bound is $\sigma=0.003$. See the above cited references for details about how to compute it.

Then, if an analogue development to Theorem 1 is carried out for TS models (adding, however, the Lipschitz-bound construction), the SOS results, as a particular case, get converted to the classical LMIs (basically, similar to the ones reported in $[8,9]$ ) given in the following corollary (details omitted for brevity):

Corollary 1. If the quadrotor's TS model (8) is used and non-polynomial fuzzy observer gains $L_{i}$ are to be designed, the observer is stable if the following LMI problem is feasible for $\alpha>0$ fixed and $i=1, \ldots, 8$ :

Minimize $\gamma$ such that

$$
P \succ 0
$$

$$
\begin{gathered}
{\left[\begin{array}{ccccc}
P-D^{T} D-\tau_{1} \sigma^{2} I & (*) & 0 & 0 & 0 \\
P \mathcal{A}_{i}-H_{i} C & P & (*) & (*) & P \\
0 & T_{s} Q^{T} P & \gamma I & 0 & 0 \\
0 & -R^{T} H_{i}^{T} & 0 & \gamma I & 0 \\
0 & P & 0 & 0 & \tau_{1} I
\end{array}\right] \succ 0} \\
{\left[\begin{array}{ccc}
\exp \left(-2 \alpha T_{s}\right) P-\tau_{2} \sigma^{2} I & (*) & 0 \\
P \mathcal{A}_{i}-H_{i} C & P & P \\
0 & P & \tau_{2} I
\end{array}\right] \succ 0} \\
\mathcal{A}_{i}^{T} P-C^{T} H_{i}^{T}+P \mathcal{A}_{i}-H_{i} C \succ 0
\end{gathered}
$$

where, $\tau_{1}>0$ and $\tau_{2}>0$ are Lagrange multipliers, $\gamma>0$, and the fuzzy observer gains can be obtained by $L_{i}=P^{-1} H_{i}$.

Furthermore, if the quadrotor's linearized model (A.1) is used $\left(\mathcal{A}_{i}=\mathcal{A}\right)$, the wellknown linear observer $L$ design (with the same criteria) trivially results from setting $\sigma=0$ in the above corollary. In the linear case, (23) state that poles must lie in the right-half plane (of course, inside the circle of radius $\exp \left(-\alpha T_{s}\right)$ from $(22)$ ).

\section{Design compromises in practice}

There are some issues to be discussed in order to obtain acceptable responses in practice from the above observer design techniques, addressed next.

\subsection{Disturbance rejection vs. decay trade-off}

In many practical cases, such as the application here discussed, there is a tradeoff between different relevant aspects:

- Performance, i.e., trying to maximize decay-rate parameter $\alpha$ in (15) for fast convergence from nonzero initial conditions. This point is very important from the tracking point of view problem.

- Worst-case disturbance rejection, i.e., robustness, as $\mathcal{H}_{\infty}$ bounds can be understood as robustness margins to unstructured time-varying uncertainty via the small-gain theorem [14]. 
- Actual-case disturbance rejection (i.e., actual mean error performance with the random noises, operating ranges and modelling errors of a test experiment).

Note that $\mathcal{H}_{\infty}$ disturbance rejection design is minimizing the effect of the "worstcase" disturbance (which can be either an external disturbance or a modeling error). However, it does not directly minimize the effect of "actual" disturbances (particularly, zero mean random noise).

From the above considerations, a better $\mathcal{H}_{\infty}$ attenuation bound (in the design phase) may result in, for instance, a larger accumulated integral squared error index (ISE) in a particular experiment (compared to an alternative design with theoretically worse $\mathcal{H}_{\infty}$ bound).

Moreover, in order to minimize the effect of the worst-case disturbance, the obtained gains may be very small so achieved decay performance is very bad. On the other hand, decay-rate optimization does not take into account the affordable amount of risk if the worst-case disturbance appears. Also, desired decay-rate performance is very related to the amount of random noise in sensor readings.

Figures 2(a) and 2(b) illustrate the above-discussed extremal cases ${ }^{4}$. For instance, with a noisy sensor, the optimal $\mathcal{H}_{\infty}$ estimated speeds are smooth (noise is very-well filtered) but with an unacceptable error from tracking point of view (Figure 2(a)). If a fast decay rate is required, the estimated speeds are also useless because there is a high amount of accumulated error due to noise spikes (Figure 2(b)).

Therefore, as there is a trade-off, there is no single "optimal" observer design, but a collection of optimal ones (the multicriteria Pareto front $[16,17]$ ). For instance, the Pareto front can be built by; $(a)$ providing the fastest decay rate for a given robustness bound, $(b)$ conversely, the better robustness bound for a given decay rate or $(c)$ a weighted combination of both following some importance criteria.

Hence, it's a choice of the end-user where to lie in the tradeoff: in practice both extreme designs may be useless (Figure 2) whereas an intermediate one (Figure 3) will be satisfactory ${ }^{5}$ for the "actual case" performance.

The above ideas suggests the following methodology in order to select, in practice, a particular observer:

I. As the actual disturbances, model errors and the to be tracked signal bandwidth in practical operation are not known with precision at design time, obtain a whole Pareto-front for multicriteria $\mathcal{H}_{\infty}$ plus decay-rate settings.

II. Carry out a representative experiment and collect input-output data.

III. Test the different observers in the Pareto front over the same data (single experiment from step II).

IV. Evaluate achieved performance (see details on the particular setting for the 3DoF system in Section 5.2.2).

V. Choose the design which has achieved the best experimental performance with the "actual case" disturbances and modelling errors.

In this way, the "practically" optimal solution is selected from a set of multiple "theoretically" optimal ones.

\footnotetext{
${ }^{4}$ Data come from the actual experimental platform, but the discussion in this section applies to more general settings.

${ }^{5}$ The referred figures have been obtained with the experiments and datasets discussed in Section 5.
} 


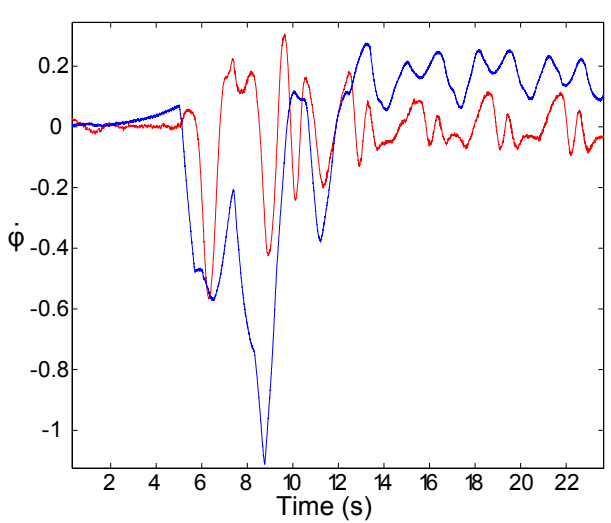

(a) Slow decay (lowest $\mathcal{H}_{\infty}$ bound)

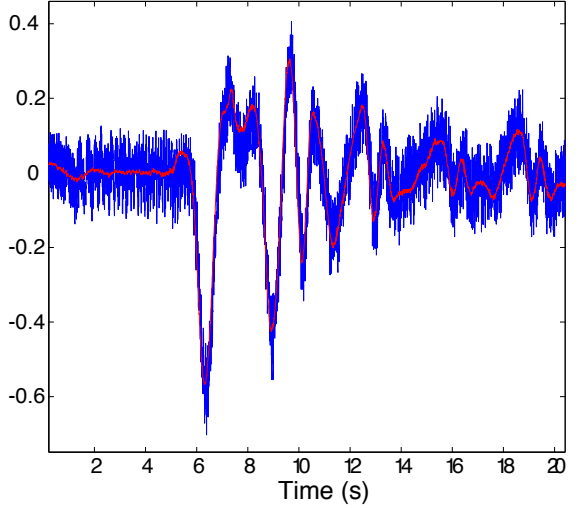

(b) Spiky estimation (fast decay)

Figure 2: Useless speed estimates in the Pareto front.

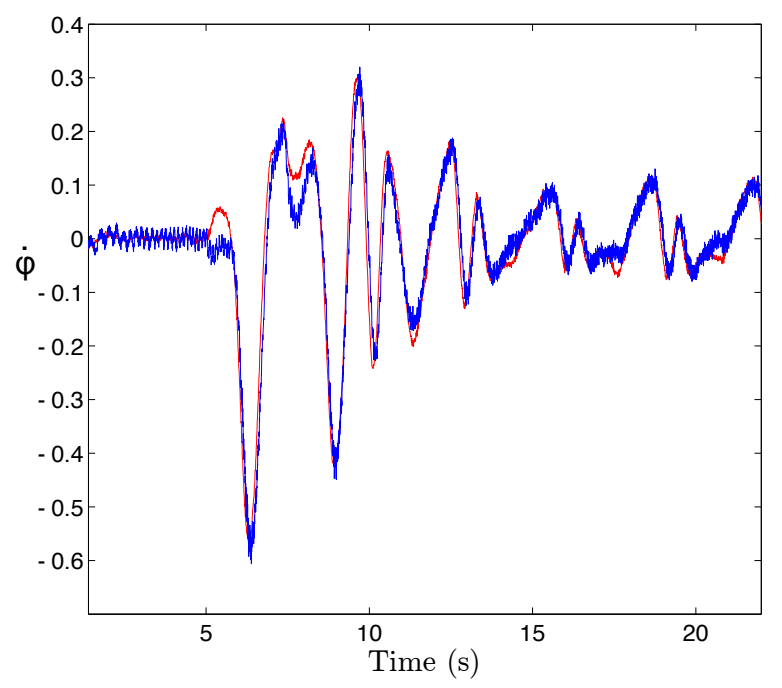

Figure 3: Acceptable speed estimates.

\subsection{Choice of disturbance size parameters}

Even if the presented results are correct in theory, there is no clear rule on how to choose observer design parameters $Q, R, D$. Indeed, the main idea is that the resulting time response of the observer error will depend on:

- the proportion between process noise/model error $Q$ and sensor noise/modelling error $R$.

- the accuracy of the model (expecting polynomial models to be more accurate than plain linearized ones). 
- the relative proportions between the elements of $D$ : giving more weight to some position or speed errors trades off a larger error for the less weighted variables.

- the decay-rate parameter discussed above.

For simplicity, from the above considerations, it is suggested to choose simple structures for the design matrices (such as diagonal), and tune actual performance of the observer via the decay-rate parameter instead of devoting too much time in modifying such matrices.

\section{Experimental results}

The above results have been experimentally tested on the platform described in Section 2.

The objectives of the experiment are; (a) showing that, with actual experimental data, reasonable solutions regarding the performance/robustness are achieved with the proposed polynomial design and (b) showing that the use of polynomial techniques dominate non-polynomial ones based on fuzzy TS models or on linearised models.

The expected disturbance, modelling errors and noise sizes on this application, jointly with considerations discussed on Section 4, made us select the following scaling matrices in order to run the optimization problem:

$$
Q=\left[\begin{array}{ccc}
0 & 0 & 0 \\
0.02 & 0 & 0 \\
0 & 0 & 0 \\
0 & 0.02 & 0 \\
0 & 0 & 0 \\
0 & 0 & 0.02
\end{array}\right] \quad R=\left[\begin{array}{ccc}
0.01 & 0 & 0 \\
0 & 0.01 & 0 \\
0 & 0 & 0.01
\end{array}\right]
$$

Indeed, by definition process disturbances on the speed dynamic equations do not exist. The constant weighting matrix D in (17) is chosen as follows

$$
D=\operatorname{diag}(0.001,0.1,0.001,0.1,0.001,0.1) ;
$$

in order to take into account speed estimation errors as the objective of the experiments.

A Pareto front with different decay rates, obtaining different suboptimal $\mathcal{H}_{\infty}$ norm bounds, is made for different observer designs (linear, TS and polynomial), being $\left[y_{k}-\right.$ $\left.C \hat{x}_{k}\right]$ the output estimation error at sample $k$.

Note that the decay-rate parameter is used to tune the aggressiveness of the observer instead of the manipulation of the disturbance-size design weights ${ }^{6}$.

The compared observer design strategies were:

- [LM-D]: $\hat{x}_{k+1}=\mathcal{A} \hat{x}_{k}+\mathcal{B} u_{k}-L\left[y_{k}-C \hat{x}_{k}\right]$, classical linear observer design with linearized model (Corollary 1 with $\mathcal{A}_{i}=\mathcal{A}, \mu=0$ ). Without theoretical guarantees in practice (only for infinitesimally small disturbances and initial conditions on state and estimated state).

\footnotetext{
${ }^{6}$ Actually, quite a few other values for those matrices were tested, but they are not reported because results are very similar to the ones presented (once the suitable Pareto-front decay-rate tuning is accounted for).
} 
- $[\mathbf{L M}-\mathbf{A}]: \hat{x}_{k+1}=\sum_{i=1}^{2} \mu_{i} \mathcal{A}_{i}\left(\hat{x}_{k}\right)+\mathcal{B}\left(u_{k}\right)-L\left[y_{k}-C\left(\hat{x}_{k}\right)\right]$, analizes the above LM-D obtained linear gain with the actual fuzzy polynomial model (Theorem 1 with fixed $\left.L_{i}\left(y_{k}, \hat{x}_{k}\right)=L\right)$. This yields a posteriori guaranteed performance in all operating region.

- $\left[\right.$ TS-8GD]: $\hat{x}_{k+1}=\sum_{i=1}^{8} \mu_{i}\left(\mathcal{A}_{i} \hat{x}_{k}-L_{i}\left[y_{k}-C \hat{x}_{k}\right]\right)+\mathcal{B} u_{k}$, optimal LMI design with fuzzy Takagi-Sugeno model (Corollary 1 ) and 8 fuzzy observer gains $L_{i}$. Guaranteed a priori performance in all operating region.

- $\left[\right.$ TS-1GD]: $\hat{x}_{k+1}=\sum_{i=1}^{8} \mu_{i}\left(\mathcal{A}_{i} \hat{x}_{k}-L\left[y_{k}-C \hat{x}_{k}\right]\right)+\mathcal{B} u_{k}$, optimal LMI design with fuzzy TS model (Corollary 1 ) and one observer gain $L_{i}=L$. Guaranteed a priori performance in all operating region.

- $[\mathbf{T S}-1 \mathbf{G A}]: \hat{x}_{k+1}=\sum_{i=1}^{2} \mu_{i} \mathcal{A}_{i}\left(\hat{x}_{k}\right)+\mathcal{B}\left(u_{k}\right)-L\left[y_{k}-C\left(\hat{x}_{k}\right)\right]$, analizes the above obtained single-gain observer with the fuzzy polynomial model (Theorem 1 with fixed $\left.L_{i}\left(y_{k}, \hat{x}_{k}\right)=L\right)$. A posteriori guaranteed performance in the operating region.

- $[\mathbf{S O S - 2 G}]: \hat{x}_{k+1}=\sum_{i=1}^{2} \mu_{i}\left(\mathcal{A}_{i}\left(\hat{x}_{k}\right)-L_{i}\left[y_{k}-C\left(\hat{x}_{k}\right)\right]\right)+\mathcal{B}\left(u_{k}\right)$, optimal SOS design with fuzzy polynomial model (Theorem 1 ), and 2 non-polynomial ${ }^{7}$ observer gains. Guaranteed a priori performance in all operating region.

Computational cost. Note that the theorems in this paper are evaluated off-line, so when computational requirements are discussed, they refer to memory and CPU of the workstation with MATLAB ${ }^{\circledR}$ code carrying out sum of squares optimizations in the observer design phase. Such optimizations are not needed in on-line operation, but only a very simple direct evaluation of (9) once the coefficients of $L$ are fixed. Such on-line evaluations can be easily encoded on a few lines of code on any low-range industrial control computer.

In the off-line gain computation, with the selected quadrotor case, Table 1 shows the demanded computational resources on a machine with Windows ${ }^{\circledR} \mathrm{XP}$, Intel Pentium ${ }^{\circledR} \mathrm{III}$ at $640 \mathrm{MHz}$ and $512 \mathrm{Mb}$ of RAM. Only the two more demanding alternatives (TS-8GD and SOS-2G with degree of multipliers $s, q$ equal to 2 ) are shown for brevity. The results were obtained using MATLAB ${ }^{\circledR}$ 6.5 R13, YALMIP R20110318 and SeDuMi 1.21.

\begin{tabular}{|c|c|c|c|c|}
\hline & Problem size & RAM & $\begin{array}{c}\text { YALMIP } \\
\text { time }\end{array}$ & $\begin{array}{c}\text { Solver } \\
\text { time }\end{array}$ \\
\hline SOS-2G & $10262 \times 1180$ & $140 \mathrm{Mb}$ & $31.48 \mathrm{~s}$ & $20.05 \mathrm{~s}$ \\
\hline TS-8GD & $11596 \times 168$ & $4 \mathrm{Mb}$ & $2.17 \mathrm{~s}$ & $13.02 \mathrm{~s}$ \\
\hline
\end{tabular}

Table 1: Approximate computational resources for design.

\subsection{Pareto-front results}

The results for the six different strategies are shown on Table 2. Some important conclusions can be extracted:

\footnotetext{
${ }^{7}$ Polynomial techniques presented on Section 3 allow to design polynomial observer gains $L_{i}(y, \hat{x})$. However, in this quadrotor particular case the use of polynomial terms in observer gains didn't seem to obtain better results than those with constant gains $L_{i}$, above reported.
} 


\begin{tabular}{|c|c|c|c|c|c|c|}
\hline$\alpha$ & $\mathbf{1 . 5}$ & $\mathbf{6}$ & $\mathbf{1 0}$ & $\mathbf{1 4}$ & $\mathbf{1 8}$ & $\mathbf{2 2}$ \\
\hline LM-D & 1.628 & 6.45 & 11.06 & 15.89 & 20.9 & 26.09 \\
\hline LM-A & 22.37 & 39.44 & 31.34 & 30.64 & 33.07 & 36.93 \\
\hline TS-8GD & 13.94 & 30.64 & 50.23 & 73.95 & 102.87 & 138.65 \\
\hline TS-1GD & 14.89 & 32.63 & 52.87 & 77.35 & 107.25 & 144.46 \\
\hline TS-1GA & 9.708 & 22.3 & 35.3 & 49.71 & 65.65 & 83.21 \\
\hline SOS-2G & 5.986 & 9.915 & 14.36 & 19.18 & 24.26 & 29.57 \\
\hline
\end{tabular}

Table 2: Set of optimal solutions in the Pareto-front sense, with different approaches.

- LM-D performances (row 1) assume that the process is linear or the operation region is infinitesimal. Hence, they are menaningless, overly optimistic. LM-A (row 2) evaluates the design (a posteriori) in the non-infinitesimal region of study with polynomial models, and results are more meaningful (markedly worse, as expected).

- Performances obtained with the TS model and 8 fuzzy gains (TS-8GD, row 3) dominate those obtained with single gain (TS-1GD, row 4), as intuitively expected. However the improvement of using fuzzy observer gains is not very significant, so the use of only single gain is justified in the present application.

- Valid TS designs (TS-8GD, TS-1GD) are very conservative due to the need of fulfilling the Lipschitz condition (19). This can be noted by comparing them with the fuzzy polynomial design results (SOS-2G), even in an a posteriori analysis (TS-1GA).

- TS designs outperform linear ones for low decay requirements (compare rows 2 and 5 on Table 2). However, for high decays, the need of ensuring Lyapunov constraints with the Lipschitz bound (19) seems to make the TS design very conservative.

- Using the SOS-2G design (row 6), the worst-case disturbance attenuation is always better than the obtained with all other LMI strategies which ensure guaranteed performance for the same decays (i.e., rows 2 to 5 ). Hence, the theoretical solutions of the SOS strategy dominate linear and TS ones in the Pareto sense, as intuitively expected.

Based on the above considerations, the best chosen design will be the fuzzy polynomial model and SOS observer design approach (Table 2, SOS-2G). The theoretical results must, however, be confronted to actual experimental performance, as done next.

\subsection{Experimental evaluation of final design}

Considering the above compromises, this section presents the final chosen observer striking a good performance/robustness balance for this application and evaluates it on the experimental platform. 


\subsubsection{Data generation.}

With the objective of validating the SOS proposed approach, the system has been subjected to an excitation achieving large enough angular speeds for the nonlinear terms to be significant. Hence, a sinusoidal excitation was introduced in $\psi$ from second 5 till 60 and a reference in $\theta$ and $\phi$ changes every 30 seconds from 10 to -10 degrees to an underlying low-gain stabilizing PI controller, providing excitation in these degrees of freedom. The collected input-output data appear in Figure 4.

The initial conditions were close to the linearization point, and in the first 5 seconds no input excitation has been applied. The set of data has been obtained during a device maneuver using a direct low-noise encoder. This data confirms that the system states satisfy the bounds from Section 2.

The objective is to check performance of observer designs in a demanding environment (otherwise, everything works perfectly if sensors are very good). In order to do that, the encoder signals have been intentionally corrupted with random noise (variance $0.001 \mathrm{rad}$ ) plus a chirp sinusoidal signal (amplitude $8 \cdot 10^{-4} \mathrm{rad}$ and frequency varying between 0.01 and $10 \mathrm{~Hz}$ in cycles of $5 \mathrm{sec}$ ) simulating a deterministic decalibration.

\subsubsection{Evaluation of observer performance}

Given the Pareto-front designs on Table 2, the problem now is selecting one of them as the chosen design for the application. Let us follow the methodology in Section 4. In order to evaluate observer performance, a "precise" state trajectory is required. With that trajectory, actual observer performance will be evaluated in terms of integral square error (ISE).

The direct low-noise encoder measurements have been used for that task. The position estimates are precise, but, as there is no direct access to the speed state variables, a noncausal zero-phase filter (filtfilt in MATLAB ${ }^{\circledR}$ with $0.5 /\left(1-0.5 z^{-1}\right.$ ) in forward and reverse time, plus further noncausal numerical differentiation $\left(z-z^{-1}\right) /\left(2 T_{s}\right)$ in the speed coordinates) has been used to compute [18] (off-line) a target "actual" value of speeds from clean position measurements (data in Figure 4). The resulting data have been assumed to be the "true" speeds to which observers should converge (note that observers are under the constraints of dealing with noise and being causal).

Evaluating the observer ISE, we finally selected the options with decay rate of $\alpha=18$ : it offered a good enough response from tracking point of view and a reduced ISE compared to other Pareto-front candidate solutions.

\subsubsection{Adopted solution}

Let us compare the LM-A option (designed with linearised model) which, in theory, gives guaranteed performance $\left(\alpha=18, \mathcal{H}_{\infty}=33.07\right)$, with the fuzzy TS single-gain design TS-1GA $\left(\alpha=18, \mathcal{H}_{\infty}=65.65\right)$ and the polynomial SOS-2G design $\left(\alpha=18, \mathcal{H}_{\infty}=\right.$ 24.264). Note that, thanks to the polynomial techniques, the SOS designs are more robust than the linear or TS ones from a theoretical point of view (lower $\mathcal{H}_{\infty}$ bound for the same decay rate). The selected observer designs are tested and compared on the quadrotor. The obtained gains are: 

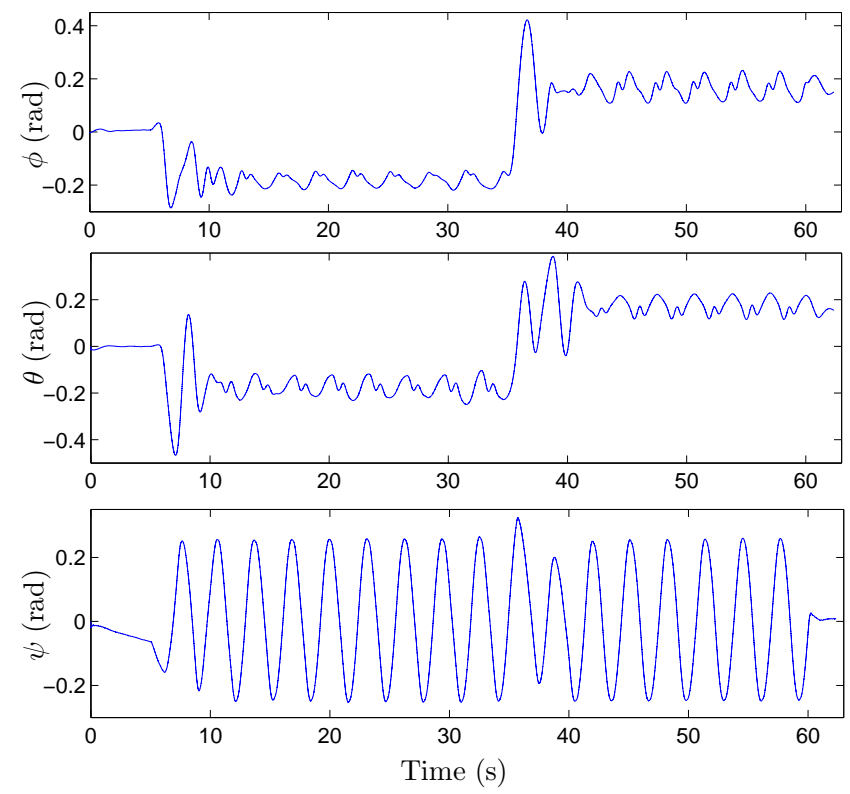

(a) Measurements
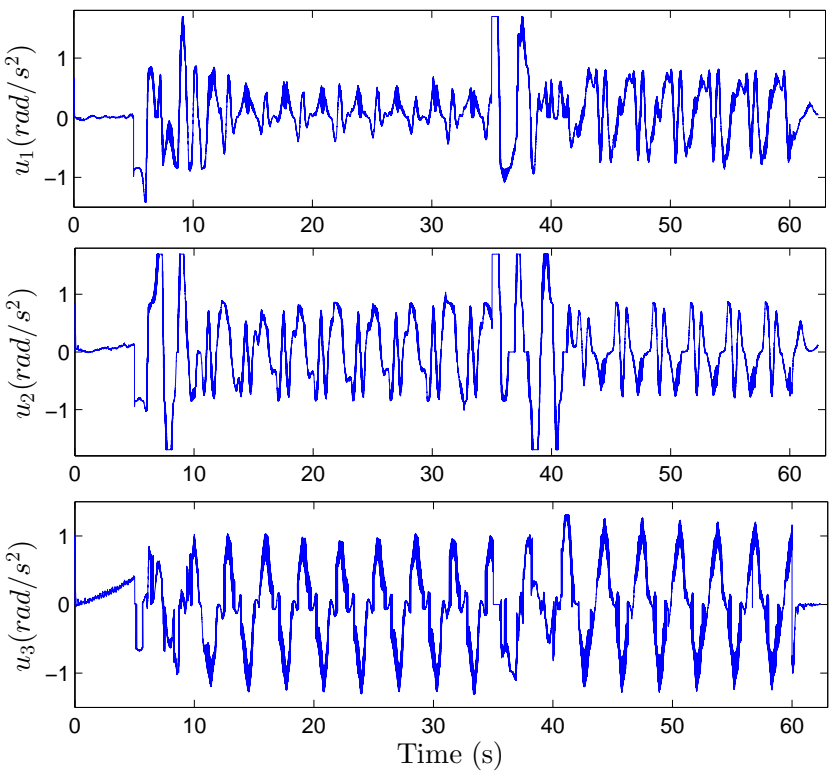

(b) Excitations

Figure 4: Collected data 


$$
\begin{aligned}
L_{L M-A}= & {\left[\begin{array}{ccc}
0.713 & 0 & 0 \\
11.253 & 0 & 0 \\
0 & 0.713 & 0 \\
0 & 11.253 & 0 \\
0 & 0 & 0.713 \\
0 & 0 & 11.253
\end{array}\right] L_{T S-1 G A}=\left[\begin{array}{ccc}
1.163 & 0 & 0 \\
52.013 & 0 & 0 \\
0 & 1.163 & 0 \\
0 & 52.013 & 0 \\
0 & 0 & 1.191 \\
0 & 0 & 53.61
\end{array}\right] } \\
L_{1_{S O S-2 G}} & =\left[\begin{array}{ccc}
0.73 & -0.062 & 0 \\
12.51 & -0.889 & 0 \\
0.062 & 0.73 & 0 \\
0.889 & 12.51 & 0 \\
0 & 0 & 0.82 \\
0 & 0 & 14.238
\end{array}\right] L_{2_{S O S-2 G}}=\left[\begin{array}{ccc}
0.73 & 0.062 & 0 \\
12.51 & 0.889 & 0 \\
-0.062 & 0.73 & 0 \\
-0.889 & 12.51 & 0 \\
0 & 0 & 0.82 \\
0 & 0 & 14.238
\end{array}\right]
\end{aligned}
$$

The following evaluates how the theoretical advantage translates into experimental behaviour.

The accumulated integral square errors (ISE) are shown in Figure 5. The LM-A and TS-1GA observers have the same decay rate performance as the optimal SOS-2G. However, the improvement of the SOS polynomial design with respect to the alternatives is confirmed in this experiment (12.5\% less ISE than LM-A and, roughly, less than half than TS-1GA). Note that the non-measurable premise TS-1GA setup ( $\sigma=0.003)$ results in an overly conservative setup whose theoretical performance and experimental ISE is far higher than the other setups ${ }^{8}$.

In summary, the results show that:

- The polynomial methodology clearly outperforms the TS design with unmeasurable premises TS-1GA.

- The polynomial setup obtains the best theoretically guaranteed worst-case $\mathcal{H}_{\infty}$ bound (both in the a priori formal design and in the a posteriori stability analysis step), see Table 2 .

- In actual-case performance the linear-only and polynomial observer setups achieve similar performance, beating the TS observer ${ }^{9}$. Note also that this experiment is likely not the "worst case" situation.

After the above confirmation that fuzzy polynomial techniques outperform TS and linear results, a detail of the time response with the finally chosen solution is shown in Figure 6, where roll dynamics has been omitted because there exist symmetry with pitch and, therefore, results are similar. The temporal evolution shows that the SOS-2G observer design can follow the real states without a high noise amplitude.

\footnotetext{
${ }^{8}$ Note, however, that TS-1GA and the SOS-2G are able to ensure validity of the design a priori: the underlying assumptions in the linear case do not actually hold in the experimental setting and its validity must be proved a posteriori (LM-A), as discussed before, so linearised theoretical and actual performance are a matter of "pure chance".

${ }^{9}$ In [8] the TS $\sigma=0.003$ observer outperformed the linear observer; however, that was due to the fact that the actual implementation of the observer used the linear model both in the "prediction" step $x_{k+1}=A x_{k}+B u_{k}$ and in the correction one $L\left[y_{k}-C \hat{x}_{k}\right]$. In the work presented here, the full nonlinear model $x_{k+1}=f\left(x_{k}, u_{k}\right)$ has been used in the prediction step, avoiding the unnecessary introduction of such linearization error. This produces, a more accurate linear observer performance than in [8].
} 


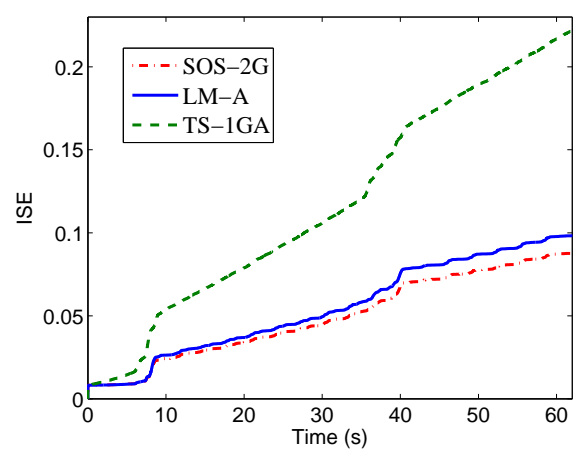

(a) Pitch speed ISE

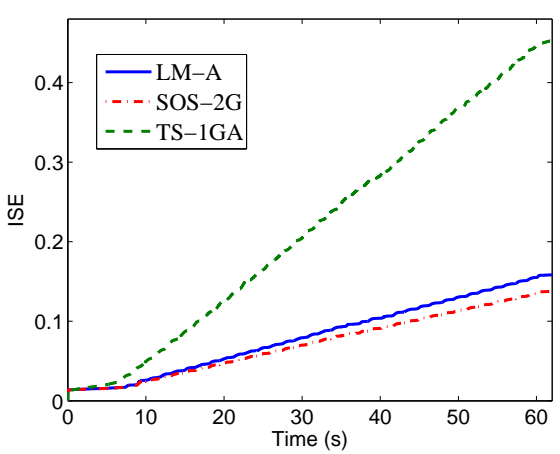

(b) Yaw speed ISE

Figure 5: Experimental ISE computation

\section{Conclusions}

In this paper, a discrete-time polynomial observer design for state estimation of an electromechanical plant with fuzzy polynomial model is presented. The design setting is multiobjective: both decay rate and $\mathcal{H}_{\infty}$ conditions are used. Fuzzy TS designs (and, of course, linearized time invariant ones) can be considered as particular cases of the proposed methodology.

The obtained observer has been implemented and checked in an experimental quadrotor platform, comparing with the fuzzy TS and classical linear designs. The results clearly show that a mixed $\mathcal{H}_{\infty} /$ decay design is needed, in order to achieve observers which are both fast and have reasonable worst-case attenuation guarantees. Furthermore, it is shown that linear and TS approaches have worse theoretical performance guarantees in a polynomial system. Obviously, the possible improvements of polynomial approaches need a model accurate enough so that the linearization errors (which polynomials avoid) contribute significantly to the overall uncertainty.

In actual experimental data, polynomial-based designs achieved a similar behaviour to linear ones when close to the linearization point and a $12 \%$ lower integral square error when operating in high-speed trajectories away from it. However, the amount of conservatism introduced in the TS design with non-measurable premises makes its performance decrease, being even worse than the obtained by the linear observer. Importantly, the proposed techniques allow for theoretical guarantee of performance, which is not the case in the naive extrapolation of linearised designs far from the equilibrium point.

\section{References}

[1] K. Tanaka, H. O. Wang, Fuzzy control systems design and analysis: a linear matrix inequality approach, 2nd Edition, Wiley-Interscience publication, John Wiley and Sons, 2001.

[2] A. Sala, C. Ariño, Polynomial fuzzy models for nonlinear control: A Taylor series approach, IEEE Transactions on Fuzzy Systems 17 (2009) 1284-1295.

[3] G. Chesi, Estimating the domain of attraction for non-polynomial systems via LMI optimizations, Automatica 45 (2009) 1536-1541. 


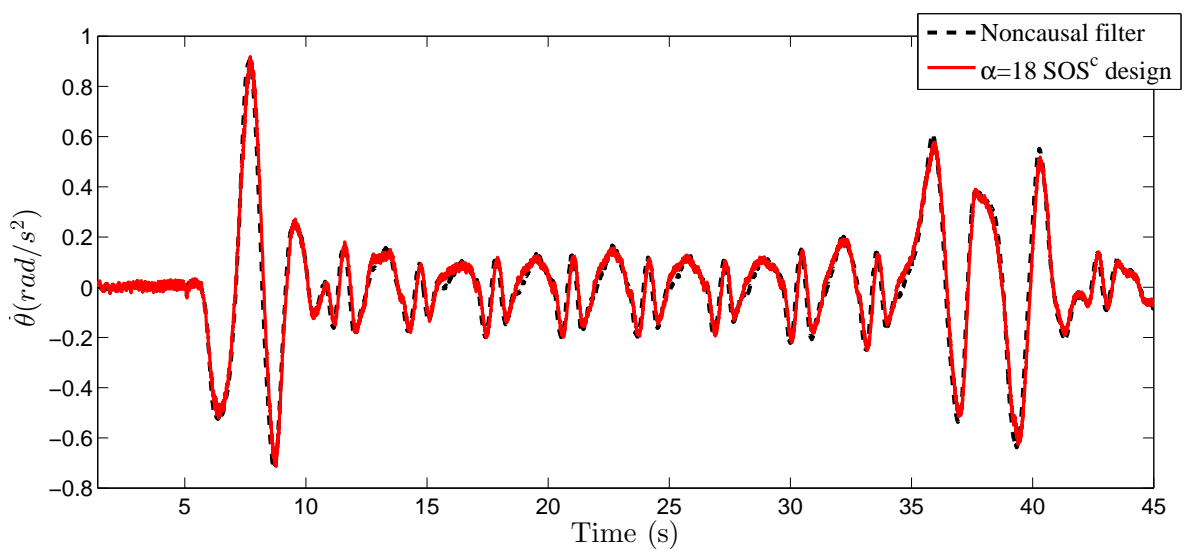

(a) Pitch estimated speed

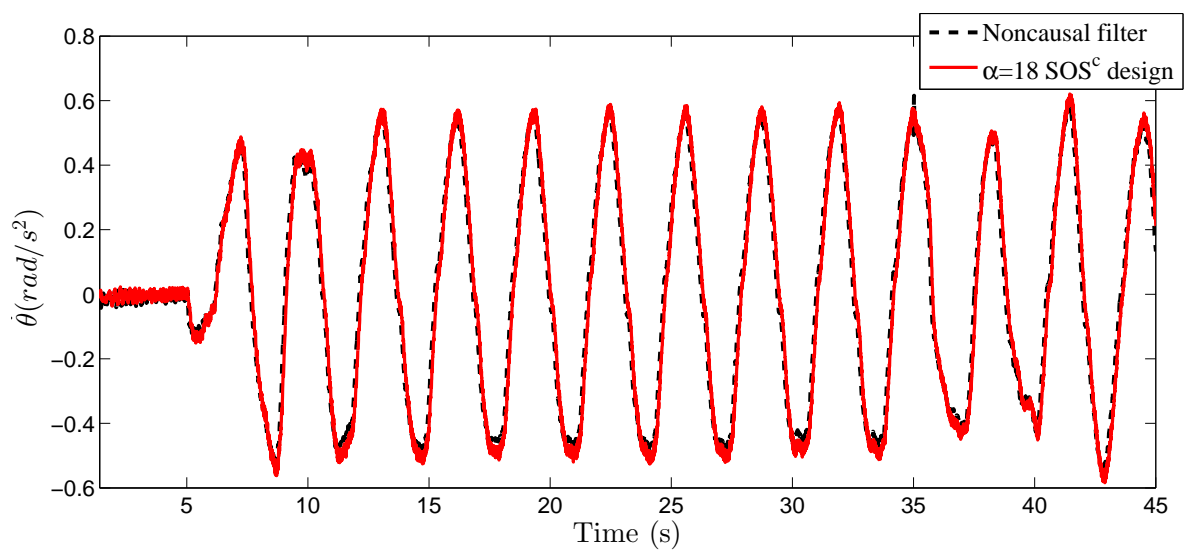

(b) Yaw estimated speed

Figure 6: Speed estimation with causal observer versus noncausal filter.

[4] S. Delrot, T. M. Guerra, M. Dambrine, F. Delmotte, Fouling detection in a heat exchanger by observer of takagisugeno type for systems with unknown polynomial inputs, Engineering Applications of Artificial Intelligence 25 (8) (2012) 1558 - 1566.

[5] C. Ariño, E. Pérez, A. Sala, Guaranteed cost control analysis and iterative design for constrained Takagi-Sugeno systems, Engineering Applications of Artificial Intelligence 23 (8) (2010) 1420 - 1427.

[6] J. Löfberg, Pre- and post-processing sum-of-squares programs in practice, IEEE Transactions on Automatic Control 54 (5) (2009) 1007-1011.

[7] T. Bouarar, K. Guelton, N. Manamanni, Robust non-quadratic static output feedback controller design for takagi-sugeno systems using descriptor redundancy, Engineering Applications of Artificial Intelligence 26 (2) (2013) $739-756$.

[8] Z. Lendek, A. Berna, J. Guzmán-Giménez, A. Sala, P. García, Application of T-S observers for state estimation in a quadrotor, in: Proc. of IEEE Con. on Decision and Control and European Control Conf. (CDC-ECC), Orlando, FL, USA, 2011, pp. 7530-7535.

[9] Z. Lendek, T. M. Guerra, R. Babuška, B. De Schutter, Stability Analysis and Nonlinear Observer Design using Takagi-Sugeno Fuzzy Models, Studies in Fuzziness and Soft Computing, Springer, 2010.

[10] Quanser, 3D Hover System manual, Avaliable on http://www.quanser.com (july 2011). 
[11] S. Bouabdallah, Design and control of quadrotors with application to autonomous flying, Ph.D. thesis, Ecole Polytechnique Federale de Lausanne (Switzerland) (2007).

[12] W. J. Rugh, J. S. Shamma, Research on gain scheduling, Automatica 36 (10) (2000) $1401-1425$.

[13] G. Chesi, On the gap between positive polynomials and sos of polynomials, Automatic Control, IEEE Transactions on 52 (6) (2007) $1066-1072$.

[14] H. K. Khalil, Nonlinear Systems, 3rd Edition, Prentice Hall, 2002

[15] C. Scherer, S. Weiland, Linear matrix inequalities in control, Notes for a course of the Dutch Institute of Systems and Control., 2004.

[16] R. Marler, J. Arora, Survey of multi-objective optimization methods for engineering, Structural and Multidisciplinary Optimization 26 (2004) 369-395.

[17] K. Miettinen, Nonlinear multiobjective optimization, Kluwer Academic Publishers, Boston, 1999.

[18] R. Merry, M. van de Molengraft, M. Steinbuch, Velocity and acceleration estimation for optical incremental encoders, Mechatronics 20 (1) (2010) $20-26$.

[19] J. F. Sturm, Using sedumi 1.02, a matlab toolbox for optimization over symmetric cones, Optimization Methods and Software 11 (1-4) (1999) 625-653.

[20] S. Boyd, L. E. Ghaoui, E. Feron, V. Balakrishnan, Linear matrix inequalities in system and control theory, no. 15 in SIAM studies in applied mathematics, SIAM, 1994.

[21] C. W. Scherer, C. W. J. Hol, Matrix sum-of-squares relaxations for robust semi-definite programs, Mathematical Programming 107 (1-2) (2006) 189-211.

[22] G. Stengle, A nullstellensatz and a positivstellensatz in semialgebraic geometry, Mathematische Annalen 207 (2) (1974) 87-97.

\section{Appendix A. Nonlinear model}

The non-linear model of platform (1) is presented in [11] with the variables and parameters shown in Table A.3.

\begin{tabular}{llll} 
Sym & Meaning & Type & Unit \\
\hline$\phi$ & Roll angle & Measured & $\mathrm{rad}$ \\
$\dot{\phi}$ & Roll angular velocity & Estimated & $\mathrm{rad} / \mathrm{s}$ \\
$\theta$ & Pitch angle & Measured & $\mathrm{rad}$ \\
$\dot{\theta}$ & Pitch angular velocity & Estimated & $\mathrm{rad} / \mathrm{s}$ \\
$\psi$ & Yaw angle & Measured & $\mathrm{rad}$ \\
$\dot{\psi}$ & Yaw angular velocity & Estimated & $\mathrm{rad} / \mathrm{s}$ \\
$V_{i}$ & Voltage to propeller $i$ & Input & $\mathrm{V}$ \\
$K_{v}$ & Transformation constant & 54.945 & $\mathrm{rad} \mathrm{s} / \mathrm{V}$ \\
$J_{r}$ & Rotators inertia & $6 \cdot 10^{-5}$ & $\mathrm{kgm}^{2}$ \\
$I_{x x}$ & Inertia X-axis & 0.0552 & $\mathrm{kgm}^{2}$ \\
$I_{y y}$ & Inertia Y-axis & 0.0552 & $\mathrm{kgm}$ \\
$I_{z z}$ & Inertia Z-axis & 0.1104 & $\mathrm{kgm}$ \\
$b$ & Thrust coefficient & $3.9351 \cdot 10^{-6}$ & $\mathrm{~N} / \mathrm{Volt}$ \\
$d$ & Drag coefficient & $1.1925 \cdot 10^{-7}$ & $\mathrm{Nm} / \mathrm{Volt}$ \\
$l$ & Distance pivot-motor & 0.1969 & $\mathrm{~m}$ \\
$m$ & Mass & 2.85 & $\mathrm{~kg}$ \\
$g$ & Gravity acceleration & 9.81 & $\mathrm{~m} / \mathrm{s}^{2}$ \\
$T_{s}$ & Sampling time & 0.005 & $\mathrm{~s}$ \\
\hline
\end{tabular}

Table A.3: Quadrotor variables and parameters

The terms $\frac{J_{r} \dot{\theta}}{I_{x x}} K_{v}\left(V_{1}+V_{3}-V_{2}-V_{4}\right)$ and $\frac{J_{r} \dot{\phi}}{I_{x x}} K_{v}\left(-V_{1}-V_{3}+V_{2}+V_{4}\right)$ denote gyroscopic effects. The terms $\frac{I_{y y}-I_{z z}}{I_{x x}} \dot{\theta} \dot{\psi}, \frac{I_{z z}-I_{x x}}{I_{y y}} \dot{\psi} \dot{\phi}, \frac{I_{x x}-I_{y y}}{I_{z z}} \dot{\theta} \dot{\phi}$, denote Coriolis effects. 
The linearized model of the quadrotor around $x=0$, discretized by Euler method (as the nonlinear model) for $T_{s}=5 \mathrm{~ms}$, is:

$$
\begin{aligned}
x_{k+1} & =\mathcal{A} x_{k}+\mathcal{B} u_{k}+T_{s} Q w_{k} \\
y_{k} & =C x_{k}+R \nu_{k}
\end{aligned}
$$

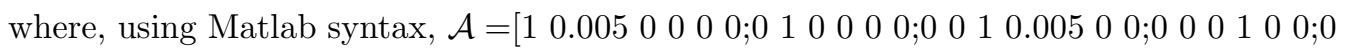

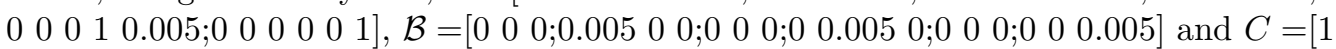

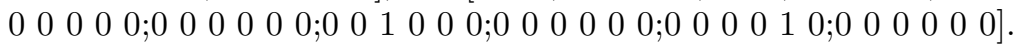

\section{Appendix B. Basic results on polynomial systems}

A polynomial $p(z)$ is said to be SOS if and only if there exist a vector of monomials $m(z)$ and a constant positive semidefinite matrix $H$ ("Gram Matrix") such that $p(z)=$ $m(z)^{T} \operatorname{Hm}(z)$. In this way, SOS decompositions of polynomials can be found using wellknown SDP software $[6,19]$ in order to search for such $H$. Note that all SOS polynomials are positive, however the reciprocal is not true [13]. For instance, the above software finds that polynomial $p(z)=z_{1}^{4}-4 z_{1}^{3} z_{2}+2 z_{1}^{3}+4 z_{1}^{2} z_{2}^{2}-12 z_{1}^{2} z_{2}+z_{1}^{2}+16 z_{1} z_{2}^{2}-8 z_{1} z_{2}+16 z_{2}^{2}$ can be written as:

$$
p(z)=\left[\begin{array}{c}
z_{1} \\
z_{2} \\
z_{1} z_{2} \\
z_{1}^{2}
\end{array}\right]^{T}\left(\begin{array}{cccc}
1 & -4 & -2 & 1 \\
-4 & 16 & 8 & -4 \\
-2 & 8 & 4 & -2 \\
1 & -4 & -2 & 1
\end{array}\right)\left[\begin{array}{c}
z_{1} \\
z_{2} \\
z_{1} z_{2} \\
z_{1}^{2}
\end{array}\right]
$$

and, as the matrix is positive definite, the Cholesky factor gives the SOS decomposition $p(z)=\left(z_{1}-4 z_{2}-2 z_{1} z_{2}+z_{1}^{2}\right)^{2}$.

The classical linear matrix inequality framework (positive-definiteness of matrices with linear expressions as elements [20]) can be also extended to the polynomial-matrix case:

Proposition 1 ([21]). Let $F(x)$ be an $N \times N$ symmetric polynomial matrix of degree $2 d$ in $x \in \mathbb{R}^{n} . F(x)$ is a matrix SOS polynomial if and only if there exist a constant matrix $Q \succeq 0$ satisfying

$$
F(x)=(I \otimes z(x))^{T} Q(I \otimes z(x)) \quad \forall x \in \mathbb{R}^{n}
$$

with $z(x(t))$ being a column vector whose entries are all monomials in $x(t)$ with degree no greater than $d$.

Local analysis. Consider a region $\Omega_{z}$ defined by polynomial inequalities $\Omega_{z}=\left\{z: g_{1}(z) \geq\right.$ $\left.0, \ldots, g_{n_{z}}(z) \geq 0\right\}$. For instance, in the quadrotor case we have $z \equiv x$ and there is only one bounding polynomial, $g_{1}(x)=R_{\max }^{2}-x^{T} x$ defining a hyper-sphere.

Lemma 1 ([22]). Consider the region $\Omega_{z}$ above. If SOS polynomials $s_{i}(z) \in \Sigma_{z}$ can be found fulfilling:

$$
p(z)-\sum_{i=1}^{n_{z}} s_{i}(z) g_{i}(z) \in \Sigma_{z}
$$

then $p(z)$ is locally non-negative in the region $\Omega_{z}$.

Polynomials $s_{i}$ are denoted as Positivstellensatz multipliers, analogous to LagrangeKKT ones in constrained optimization. 


\section{Appendix C. Proof of Theorem 1}

Condition (13) ensures positive-definiteness of the Lyapunov function, which in the linear LMI case amounts to (20). Under no disturbances, the basic decay-rate discrete condition $\Delta V=V_{k+1}-\exp \left(-2 \alpha T_{s}\right) V_{k}<0$ can be expressed as:

$$
\begin{aligned}
-\Delta V=\sum_{i=1}^{2} \mu_{i} e^{T}\left(\exp \left(-2 \alpha T_{s}\right) P-(*)^{T}\right. & P P^{-1} P \\
& \left.\left(\bar{A}_{i}(x, \hat{x})-L_{i}(\hat{x}, C(x)) \bar{C}(x, \hat{x})\right)\right) e>0
\end{aligned}
$$

Then, by using the convex-sum property and adding local information following Lemma 1 in order to make the positiveness condition of (C.1) hold only locally in the required regions, it leads to

$$
\begin{aligned}
e^{T}\left(\exp \left(-2 \alpha T_{s}\right) P-(*)^{T} P P^{-1} P\left(\bar{A}_{i}(x, \hat{x})\right.\right. & \\
& \left.\left.-L_{i}(\hat{x}, C(x)) \bar{C}(x, \hat{x})\right)\right) e-\Psi_{2 i}(x, \hat{x})>0 \quad i: 1,2
\end{aligned}
$$

being $\Psi_{2 i}(x, \hat{x})=s_{2 i}(x, \hat{x})\left(R_{\max }-x^{T} x\right)+q_{2 i}(x, \hat{x})\left(R_{\max }-e^{T} e\right)$. This is, by Schur complement, equivalent to the $i: 1,2$ convex conditions:

$$
\left[\begin{array}{cc}
\exp \left(-2 \alpha T_{s}\right) P-\Psi_{2 i}(x, \hat{x}) & (*)^{T} \\
P \bar{A}_{i}(x, \hat{x})-H_{i}(\hat{x}, C(x)) \bar{C}(x, \hat{x}) & P
\end{array}\right]>0
$$

Conditions (C.3) are fulfilled by enforcing polynomial matrices to be SOS. This can be done applying Proposition 1. In this way, (15) is obtained and $V$ is a valid Lyapunov function ensuring exponential stability of system (6).

In the disturbance case, conditions (14) are obtained by a similar procedure with the dissipation inequality $V_{k+1}-V_{k}+e_{k}^{T} D^{T} D e_{k}-\gamma^{2}\left(w^{T} w+v^{T} v\right)<0$ plus information about locality in multipliers in $\Psi_{1 i}$ (details omitted for brevity). Furthermore, if initial conditions are $x_{0}=0$ and adding from time zero till time $k$ (and letting later $k \rightarrow \infty$ ) the above discrete-time inequality results in:

$$
V\left(e_{k}\right)+e_{k}^{T} D^{T} D e_{k}-\gamma W_{k}^{T} W_{k}<0 \Rightarrow\|D e\|_{2}^{2}<\gamma\|W\|_{2}^{2}
$$

Note that $L_{i}(\hat{x}, C(x))$ is used in decay-rate conditions but $L_{i}(\hat{x}, y)$ in dissipation ones. This is because decay-rate conditions consider a noise-free dynamics in nonzero initial conditions, so $y=C(x)+R \nu$ applies for the particular case $\nu=0$.

Finally, the meaning of conditions (16) in the linear case, would be forcing the real part of the poles to be non-negative (pole-region placement results in [20]), which is actually (23). See Remark 1 for its interpretation. 